\title{
Detection rate of Pneumococcal Pneumonia using the Urine Antigen Kit BinaxNow Streptococcus Pneumoniae Test: a Prospective Cohort Study in a Tertiary Hospital in Malaysia
}

Mohamed Faisal AH, ${ }^{a}$ Petrick $\mathrm{P}^{\mathrm{b}}$ Andrea $\mathrm{YL}$ Ban, ${ }^{\mathrm{a}}$ Roslina $\mathrm{AM}^{\mathrm{a}}$

${ }^{a}$ Respiratory Unit, University Kebangsaan Malaysia Medical Centre

${ }^{b}$ Infectious Disease Unit, University Kebangsaan Malaysia Medical Centre

\begin{abstract}
Background: Pneumococcal pneumonia is the commonest type of bacterial pneumonia worldwide. In Malaysia, the reported incidence of pneumococcal pneumonia is low. This may be due to poor sensitivity of conventional methods. The use of BinaxNOW, a urinary antigen test for Streptococcus pneumoniae can aid in the diagnosis of pneumococcal pneumonia. Objective: To determine the detection rate of pneumococcal pneumonia using BinaxNOW kit and to compare this with the conventional culture method for blood and sputum. Methods: This was a prospective cohort study involving patients 18 year-old and above admitted for community-acquired pneumonia (CAP) or health-care associated pneumonia (HCAP) to UKMMC between December 2011 and June 2012. BinaxNOW test, serology for Mycoplasma, Legionella and Chlamydia and cultures from blood/sputum/other respiratory tract samples and other routine investigations were performed. Results: A total of 102 patients were recruited; $89(87.3 \%)$ with CAP, $13(12.7 \%)$ with HCAP. 57 (55.9\%) had pneumonia of unknown aetiology, 11 patients $(10.8 \%)$ had positive blood cultures, 8 patients (7.8\%) had a positive urine BinaxNOW test and 6 patients $(5.9 \%)$ had positive sputum cultures. Streptococcus pneumoniae was the commonest isolate accounting for 9 cases (8.8\%) followed by Klebsiella pneumoniae in 7 cases $(6.9 \%) .8$ out of 9 patients with positive urine BinaxNOW test had negative cultures for Streptococcus pneumoniae. One patient grew Streptococcus pneumoniae from the tracheal aspirate however the urine BinaxNOW test was negative. Conclusion: The use of urine BinaxNOW kit in UKMMC increased the detection rate of pneumococcal pneumonia in hospitalized patients.
\end{abstract}

Keywords: Streptococcus pneumoniae, community-acquired pneumonia, health-care associated pneumonia, BinaxNOW

\section{INTRODUCTION}

Community-acquired pneumonia is one of the leading causes of hospitalization. For individuals more than 65 years of age, the rates of hospitalization and death caused by pneumonia continue to increase. ${ }^{1}$ Streptococcus pneumoniae remains the commonest cause of communityacquired pneumonia in United States and Europe. It accounts for $30-40 \%$ of cases of pneumonia. ${ }^{2}$ Despite being the leading cause of pneumonia, it can only be isolated in $5-18 \%$ cases. $^{3}$ Isolation of Streptococcus pneumoniae from sputum may represent colonization, ${ }^{4}$ and invasive tests are

Corresponding author:

Mohamed Faisal bin Abdul Hamid

Respiratory Unit,

University Kebangsaan Malaysia Medical Centre

Jalan Yaacob Latif

Bandar Tun Razak

56000 Cheras, Kuala Lumpur

Malaysia

Email: arabinose@hotmail.com rarely performed. Most physicians choose to treat empirically as the organism is not easily isolated.

There is a lack of local data in Malaysia on the microbiology of community-acquired pneumonia. Liam et al. ${ }^{5}$ from University Malaya Medical Centre (UMMC) in 2001 showed that out of 127 patients hospitalized for community-acquired pneumonia, an aetiological diagnosis was achieved in 53 cases (41.7\%).

The commonest isolate was Klebsiella pneumoniae $(10.2 \%)$, followed by Streptococcus pneumoniae (5.5\%), Haemophilus influenzae (5.5\%), Mycoplasma pneumoniae (3.9\%) and Pseudomonas aeruginosa (3.9\%). Streptococcus pneumoniae was the second most common pathogen isolated using conventional methods in that study.

Hooi et al. ${ }^{6}$ identified 42 culture-positive pneumonia out of 98 patients (42.9\%) and Mycobacterium tuberculosis was the commonest pathogen being identified in $15.3 \%$ of cases, followed by Klebsiella pneumoniae (7.2\%), Pseudomonas aeruginosa (6.1\%). Streptoccocus pneumoniae was only isolated in 3 cases $(3.1 \%)$. 
Mustafa et al. ${ }^{7}$ in 2011 showed that the top three organisms detected were Streptococcus pneumoniae in $21.7 \%$ of cases, followed by Klebsiella pneumoniae (17.3\%), and Burkholderia pseudomallei (13\%). In this study multiplex real-time PCR was used. This was the first study in Malaysia to successfully show Streptococcus pneumoniae as the commonest cause of CAP.

Another study by Nazlin et al. ${ }^{8}$ in 2011 showed that immunochromatography (ICT) and PCR methods may improve the detection rate of Streptococcus pneumoniae in CAP patients compared with conventional culture. In their study, Streptococcus pneumoniae was the second commonest organism isolated (51.1\%) after Chlamydia pneumoniae (63.8\%).

Since all the studies in Malaysia using conventional methods failed to increase the detection rate of Streptococcus pneumoniae, we would like to demonstrate whether an alternative laboratory method using urinary BinaxNOW test is of additional value to the diagnostics of pneumococcal pneumonia.

\section{MATERIALS AND METHODS}

This was a prospective study carried out in Universiti Kebangsaan Malaysia Medical Centre (UKMMC), an 819-bed teaching hospital in Kuala Lumpur. The study was approved by the Research Committee, Faculty of Medicine, Universiti Kebangsaan Malaysia. The project code for this study is FF-347-2011. Patients were informed of the conduct of the study and its objectives and were recruited into the study upon a written informed consent.

Patients were hospitalized adult patients more than 18 year-old with a primary diagnosis of CAP or HCAP in the medical wards, medical high dependency ward, and ICU. The study aimed to evaluate the BinaxNOW rapid test for detection of Streptococcus pneumoniae in inpatients with CAP and HCAP in UKMMC and to compare this with conventional testing.

The inclusion criteria9,10 were (1) patients with clinical features consistent with acute lower respiratory tract infection including 2 of the following: new/worsening cough, dyspnoea, pulmonary consolidation by physical examination, pleuritic chest pain, constitutional symptoms such as arthralgia, myalgia, headache, sore throat, coryza, sweating; (2) temperature $>37.8^{\circ} \mathrm{C}$; and (3) chest radiograph showing radiographic pulmonary infiltrate consistent with pneumonia.

Patients who were excluded were (1) immunosuppressed patients [those on prednisolone (>15mg/day), azathioprine, or cyclosporine for at least 3 months prior to presentation]; (2) patients with prior history of streptococcal pneumonia within the last 3 months and were tested positive with BinaxNOW test; (3) those who received pneumococcal vaccination within the previous one week; ${ }^{11,12}$ (4) patients with hospital-acquired pneumonia and aspiration pneumonia; (5) pneumonia caused by either tuberculosis, or other possible diagnoses such as pulmonary edema, pulmonary embolism, lung cancer; ${ }^{10}$ (6) patients with neutropenic sepsis not due to pneumonia; and (7) patients who are unable to produce urine.

Recruited patients had routine investigations performed including full blood count $(\mathrm{FBC})$, renal profile (RP), liver function test (LFT), C-reactive protein (CRP), serology for atypical pneumonia (Chlamydia, Mycoplasma, and Legionella), blood culture, sputum culture, and chest radiograph as well as cultures from tracheal aspirate, bronchoalveolar lavage (BAL) and pleural aspiration when necessary.

The chest radiographs were interpreted and evaluated by in-house radiologist. Twenty millilitres $(\mathrm{mL})$ of blood were withdrawn and $10 \mathrm{~mL}$ of urine collected. Urine for BinaxNOW was collected from all patients within 48 hours of admission. Once collected, the urine sample within 2 hours of collection during working hours; if urine specimens were collected at night, they were stored at $-2^{\circ} \mathrm{C}$ and sent to the lab in an icepack the following morning.

All urine tests were tested within 24 hours of collection. The samples were allowed to equilibrate to room temperature $\left(59-86^{\circ} \mathrm{F}, 15-30^{\circ} \mathrm{C}\right)$ before the non-concentrated urine samples were tested according to the manufacturer's instructions.

\section{Statistical Analysis}

Analysis of data was performed using the statistical software product and services (SPSS) version 20.0. Cases of pneumococcal and non-pneumococcal pneumonias were compared to determine factors that favour the diagnosis of pneumococcal pneumonia while culture-negative pneumonia was excluded during the process. All numerical data were subjected to normality testing using Kolmogorov-Smirnov analysis.

Normally distributed data were expressed by mean and non-normally distributed numerical data were expressed as median + Inter-Quartile Range (IQR). Association between continuous variables was tested using non-parametric test for non-normally distributed data.

Association between two categorical data was tested using the Pearson coefficient or the Fisher's exact probability test depending on the applicability of the test. Level of significance was set at $5 \%$ ( $\alpha=$ 0.05). 


\section{RESULTS}

There were 412 admissions for pneumonia to the medical wards and intensive care unit during the period from December 2011 to June 2012. One hundred and two patients who fulfilled inclusion and exclusion criteria were recruited.

The median age was 67 years. The youngest patient was 21 year-old and the oldest was 92. Our data showed that the age distribution of study population was skewed to the left. There were 58 male patients (56.9\%) and 44 female patients (43.1\%).

There were 44 Chinese (43.1\%), 37 Malays (36.3\%), 19 Indians (18.6\%) and 2 were classified as other races $(2.0 \%)$. Of the 102 patients recruited 89 (87.3\%) had CAP and 13 had HCAP (12.7\%).

Seven patients were from ICU, 8 patients from HDW while 87 patients were from the medical wards. The median length of stay was 6 (4-10) days for CAP patients and 7 (5-9) days for HCAP patients. All patients had comorbid illness (Table I). The most common was diabetes mellitus in 39 patients (38.2\%) and the median duration of diabetes was 7 (3-10) years. The median HbA1c was $7.6 \mathrm{mmol} / \mathrm{L}$ (6.4 $-10.5)$.

Ten patients (9.8\%) died in our hospital (Table II), 6 had an aetiological diagnosis of pneumonia, (CAP, $\mathrm{n}=4$; HCAP, $\mathrm{n}=2$ ). Two patients had pneumonia due to Streptococcus pneumoniae, 2 patients had Klebsiella pneumoniae, and 1 had Staphylococcus aureus pneumonia and another had Burkholderia cepacia pneumonia.

The sputum culture was positive in 17 patients $(16.7 \%), 11$ patients $(10.8 \%)$, had positive blood cultures, 8 patients $(7.8 \%)$ had positive urine BinaxNOW, 5 patients $(5.9 \%)$ had positive tracheal aspirate. One patient $(1 \%)$ had a positive BAL and atypical pneumonia serology testing (positive for both Mycoplasma and Chlamydia) and 1 patient (1\%) had a positive pleural culture (Table III).

Candida species was isolated from the sputum culture of 11 patients (10.8\%) (Table III). These isolates were most likely a contaminant and the patients recovered without antifungal agents.
Table I: Baseline Characteristics of Study Cohort $(n=102)$

\begin{tabular}{lc}
\hline End point & $n=102(\%)$ \\
\hline Age in years & \\
& \\
Sex & \\
Male & $58(56.9)$ \\
Female & $44(43.1)$ \\
Race & \\
Malay & $37(36.3)$ \\
Chinese & $44(43.1)$ \\
Indian & $19(18.6)$ \\
& \\
Smoking status & \\
Active smoker & \\
Ex-Smoker & $17(16.7)$ \\
Never & $28(27.5)$ \\
Comorbidity & $57(55.9)$ \\
Pulmonary & \\
COPD & \\
Asthma & $17(16.7)$ \\
OSAS & $19(18.6)$ \\
Bronchiectasis & $1(1)$ \\
Old PTB & $2(2)$ \\
Diabetes Mellitus & $4(3.9)$ \\
Hypertension & $39(38.2)$ \\
Ischaemic Heart Disease & $57(55.9)$ \\
Congestive Cardiac Failure & $10(9.8)$ \\
Neoplastic Condition & $10(9.8)$ \\
Chronic Kidney Disease & $1(2 \%)$ \\
Parkinson & $1(1 \%)$ \\
Rheumatoid Arthritis & $1(1 \%)$ \\
HIV & \\
Inflammatory Bowel Disease \\
\hline
\end{tabular}

Table II: Types and Outcome of Pneumonia in UKMMC $(n=102)$

\begin{tabular}{ll}
\hline End point & $n=102(\%)$ \\
\hline $\begin{array}{l}\text { Types of pneumonia } \\
\text { CAP }\end{array}$ & $89(87.3)$ \\
HCAP & $13(12.7)$ \\
Length of Hospital Stay & \\
CAP & \\
Median ( IQR) & $6(4-10)$ \\
HCAP & \\
Median (IQR) & $7(5-9)$ \\
Outcome & \\
Discharge & $90(88.2)$ \\
At Own Risk Discharge & $1(1)$ \\
Transfer to other hospital & $1(1)$ \\
Died & $10(9.8)$ \\
CAP with aetiological diagnosis & $4(3.9)$ \\
CAP without aetiological diagnosis & $2(2)$ \\
HCAP with aetiological diagnosis & $2(2)$ \\
HCAP without aetiological diagnosis & $2(2)$ \\
\hline
\end{tabular}


By disregarding candida, the actual number of culture positive cases was $34(33.3 \%)$ and were most commonly from blood cultures.

The commonest organism detected (Table IV) was Streptococcus pneumoniae in 9 patients $(8.8 \%)$. Eight out of the 9 patients $(88.9 \%)$ were diagnosed using BinaxNOW kit only as cultures from both respiratory secretions (Sputum/Tracheal/BAL) and blood were negative. Only one of these nine patients $(11.1 \%)$ grew Streptococcus pneumoniae from tracheal aspirate, however pneumococcal BINAXNOW test in this patient was negative.

Table III: Method of Isolation of Organism

\begin{tabular}{llll}
\hline Culture & CAP, $\mathrm{n}$ & HCAP, $\mathrm{n}$ & Total, $\mathrm{n}(\%)$ \\
\hline $\begin{array}{l}\text { Negative } \\
\text { Culture }\end{array}$ & 60 & 8 & $68(66.7 \%)$ \\
\hline Positive & & & \\
Culture & & & \\
$\begin{array}{l}\text { Sputum* } \\
\text { Blood }\end{array}$ & 5 & 1 & $6(5.9)$ \\
Tracheal & 5 & 2 & $11(10.8)$ \\
Pleural & 1 & 0 & $5(4.9)$ \\
BAL & 1 & 0 & $1(1)$ \\
BINAX & 6 & 0 & $1(1)$ \\
Mycoplasma & 1 & 2 & $8(7.8)$ \\
Serology & 1 & 0 & $1(1)$ \\
Chlamydia & 1 & 0 & $1(1)$ \\
Serology & & & \\
\hline Total & 89 & 13 & $102(100)$ \\
\hline
\end{tabular}

*11 patients that grew Candida species from sputum were considered as culture negative

Table IV: Putative causative microorganisms in CAP/HCAP

\begin{tabular}{llll}
\hline Microbiology & CAP, & HCAP, & Total, \\
\hline Negative $^{*}$ & 60 & 8 & $68(66.7)$ \\
Streptococcus pneumonia & 7 & 2 & $9(8.8 \%)$ \\
Klebsiella pneumoniae & 6 & 1 & $7(6.9 \%)$ \\
Burkholderia cepacia & 3 & 1 & $4(3.9 \%)$ \\
Pseudomonas aeruginosa & 3 & 0 & $3(2.9 \%)$ \\
Staphylococcus aureus & 2 & 0 & $2(2.0 \%)$ \\
Streptococcus pyogenes & 1 & 0 & $1(1 \%)$ \\
Escherichia coli & 1 & 0 & $1(1 \%)$ \\
Streptococcus cancellatus & 1 & 0 & $1(1 \%)$ \\
Mycoplasma pneumoniae & 1 & 0 & $1(1 \%)$ \\
Chlamydia pneumoniae & 1 & 0 & $1(1 \%)$ \\
Bacillus & 1 & 0 & $1(1 \%)$ \\
MRSA & 1 & 0 & $1(1 \%)$ \\
Acinetobacter species & 0 & 1 & $1(1 \%)$ \\
Candida glabrata & 1 & 0 & $1(1 \%)$
\end{tabular}

*11 patients that grew Candida spp from sputum were considered as culture negative
The second commonest organism was Klebsiella pneumoniae in 7 patients $(6.4 \%)$. Three of them (42.9\%) had diabetes mellitus. Another emerging organism in our hospital is Burkholderia cepacia in 4 cases $(3.9 \%)$. Pseudomonas aeroginosa was isolated from 3 patients $(2.9 \%)$ and Staphylococcus aureus was isolated in 2 patients ( $2 \%)$.

From our data, we have detected 9 cases $(8.8 \%)$ of Streptococcus pneumoniae, and 8 of them were only detected by pneumococcal UAT. Their median age was 63 years $(47-73)$. Seven cases were detected among CAP patients while 2 were detected among HCAP patients. Four patients had one comorbidity; diabetes mellitus, colonic cancer, polymyositis, and HIV respectively. One patient had no comorbidites. The remaining patients had more than 2 comorbidities. One patient had diabetes mellitus, ischaemic heart disease and hypertension; another had hypertension and CKD; the third had bronchial asthma, diabetes mellitus and hypertension and the fourth had COPD, ischaemic heart disease and prostate carcinoma. Four out of 8 patients with positive BinaxNOW received antibiotics prior to admission.

One case was detected via conventional method, this patient had a positive tracheal aspirate and the assay was performed within 24 hours of admission to ICU. This patient was admitted for severe CAP and was discharged after 4 days of hospitalization. The median length of hospital stay for all pneumococcal pneumonia patients was 6 days $(5-10)$. Two patients succumbed to illness after 13 and 35 days respectively. In conclusion, the use of urine BinaxNOW kit in UKMMC increased the detection rate of pneumococcal pneumonia in hospitalized patients.

\section{DISCUSSION}

In our study population the median age of patients admitted for CAP and HCAP was 67 years old. This is similar to the average age of pneumonia patients in other western publications. ${ }^{9,10}$ However, our patients appeared to be older compared to two other Malaysian CAP studies which showed mean age of $54.7^{7}$ and $55.9^{6}$ years.

The prevalance of Streptococcus pneumoniae in Malaysia might be underestimated due to lack of proper diagnostic tools. Our study revealed a higher number of Streptococcus pneumoniae (8.8\%) compared to other organisms. By using urine BinaxNOW test, we managed to diagnose an extra 8 cases of Streptococcus pneumoniae from the culture negative group.

We also managed to diagnose 2 cases $(15.4 \%)$ of Streptococcus pneumoniae among HCAP patients. Data from other studies revealed Streptococcus pneumoniae as one of the pathogens causing HCAP accounting in $5.5-27.8 \%$ of cases. ${ }^{11-14}$ Although we have detected more Streptococcus pneumoniae 
compared to other organisms, the detection rate of Streptococcus pneumoniae by BinaxNOW test is still low compared to various studies performed overseas. A study in Spain by Andreo et al. revealed positivity rate of $16.3 \%$ in 92 patients using nonconcentrated urine. ${ }^{15}$ In that study, the total number of recruited patients was smaller compared with our study. Our detection rate of pneumococcal pneumonia is almost similar to 2 studies performed in Australia. Lauren et al. ${ }^{16}$ demonstrated prevalence of positive BinaxNOW in only 7.6\% (13 out of 170 patients). Charles et al had performed a large prospective Australian epidemiologic study of CAP aetiology, which showed a rate of $11.2 \%$ of positive BinaxNOW. ${ }^{17}$

We also compared our data with other studies in South East Asia using urine BinaxNOW kit. Two studies in Thailand found Streptococcus pneumoniae as one of the commonest organisms causing CAP. Wattanathum et al. ${ }^{18}$ found Streptococcus pneumoniae $(22.4 \%)$ and Chlamydophila pneumoniae $(16.3 \%)$ as the most frequent causative pathogens found in hospitalized patients with CAP. In our study, by using the urinary BinaxNOW, we also increased our yield of Streptococcus pneumoniae. Reechaipichitkul et al. ${ }^{19}$ found Haemophilus influenzae (31.8\%) and Streptococcus pneumoniae $(27.3 \%)$ as the commonest aetiology of CAP in the outpatient setting. Other studies conducted in India, Japan and Taiwan showed that the commonest cause of CAP was Streptococcus pneumoniae ranging from $23.8-35.8 \%$ of cases. ${ }^{20-23}$

Our low detection rate of Streptococcus pneumoniae may be reflective of our local aetiology. Excluding Mycobacterium tuberculosis, two other previous studies in Malaysia ${ }^{5,6}$ published in 2001 revealed Klebsiella pneumoniae as the commonest organism in hospitalized CAP patients although these studies were performed using conventional methods only. Another recent study in 2011 found Streptococcus pneumoniae as the commonest organism isolated using multiplex realtime PCR, ${ }^{7}$ however, the number of patients in their study population was only 49 patients.

Selection bias may be another reason why our detection rate was low as studies were only performed in hospitalised patients and might not be reflective of true epidemiology of pneumococcal pneumonia. In Thailand, the prevalence of Streptococcus pneumoniae in outpatient setting ranged from $13.3-27.3 \%$ using urinary antigen detection ${ }^{18}$ and sputum culture. ${ }^{19}$ Outpatients with less severe pneumonia were seen in the emergency department and treated as outpatient were not included in the study. This may have reduced the total number of pneumococcal pneumonia detected.

Our study protocol used non-concentrated urine for BinaxNOW based on manufacturer's instructions which may reduce the detection rate of pneumococcal pneumonia. There are studies that shows using concentrated urine increase the yield of detection of Streptococcus pneumoniae. ${ }^{10,15,24,25}$ Marcos et al. ${ }^{25}$ found that the positivity of BinaxNOW in their study increased from $109(27 \%)$ using non-concentrated urine to 154 (39\%) using concentrated urine samples. Urine samples were boiled for 5 minutes and centrifuged at $1,000 \times g$ for $15 \mathrm{~min}$. The urine was concentrated 25 -fold by selective ultrafiltration. Although it may increase the detection rate of Streptococcus pneumoniae, concentrated urine will increase the cost as well as time taken to perform this test which eventually delays the diagnosis.

We know from previous studies that BinaxNOW kit has a sensitivity ranging from $77-88 \%^{10,24-27}$ and specificity of $97-100 \% .^{10,24,27}$ However, in our study, we did not manage to check the sensitivity and specificity of this kit compared to conventional methods as all the patients with positive BinaxNOW kit had negative cultures from conventional methods. This positive BinaxNOW result was unlikely to be false positive as these patients did not receive recent pneumococcal vaccination and had no history of pneumococcal infection prior to admission. Studies have shown that pneumococcal vaccine presents sufficient antigen load to cause urinary excretion that can be detected by commercially available antigen tests but was not detected after 7 days. ${ }^{28,29}$

Blood cultures were performed for all patients in our study population and sputum cultures were done for 51 patients $(50 \%)$. One postulated reason for the negative culture results for Streptococcus pneumoniae in our study population was the inability to monitor the quantity of blood withdrawn for culture by our house-officers and thus the volume of blood obtained might had been inadequate. Other possible reasons are the use of prior antibiotic therapy, delays in sending and processing the urine samples and the inability of some patients to produce sputum.

We detected one case of pneumococcal pneumonia from an HIV infected patient using BinaxNOW kit. Although there was concern that this test may not be useful in HIV patients due to false positive result, data from other studies showed that BinaxNOW provides a credible rapid diagnostic test for pneumococcal pneumonia regardless of HIVstatus. ${ }^{30}$ Out of 8 cases of positive BinaxNOW, we were able to diagnose 4 cases $(50 \%)$ of pneumococcal pneumonia who had received antibiotic 2-5 days prior to admission. In comparison to other studies using larger study population, Gutiérrez et al. ${ }^{26}$ detected 13/104 $(12.5 \%)$ patients with positive BinaxNOW who had received antibiotics prior to admission. In another study, Lauren et $\mathrm{al}^{16}$ found $15.3 \%$ patients with positive BinaxNOW who had received antibiotics prior to admission. These studies and ours showed that this test has clinical utility in CAP patients who had received antibiotics. 
We detected one patient with a false negative BinaxNOW. We could not explain why this patient with Streptococcus pneumoniae isolated from tracheal aspirate had a negative BinaxNOW result. This patient was intubated electively due to exacerbation of bronchial asthma secondary to CAP, and tracheal aspirate was taken through the endotracheal tube (ETT). This patient responded to ceftriaxone and azithromycin and was discharged well. She did not receive any antibiotic prior to admission and the sample was collected within 48 hours of hospitalization. As described by Boulware et $\mathrm{al}^{30}$ false negative BinaxNOW occurs in about $20 \%$ of patients with pneumococcal pneumonia. Causes for false negative results include etiologic misclassification, low levels of C-polysaccharide antigen present, or sequestration of the antigen by antibodies in serum or urine as immune-complexes. The proportion of false negative results is similar to other studies. Gutierez et al. ${ }^{26}$ have found 3 patients with false negative BinaxNOW (23.1\%) while Domínguez et al. ${ }^{24}$ found 10 patients (19.5\%).

The early detection of pneumococcal pneumonia may avoid unnecessary usage of broad spectrum antibiotics. It may also avoid additional usage of macrolides of atypical organism coverage. In Malaysia there is a significant rise in macrolide resistance among Malaysian pneumococcal isolates based on a recent publication. ${ }^{31}$ Whether the emergence of this resistance was associated with overzealous use of macrolides needs further studies. Narrowing the spectrum of given antibiotics once pneumococcal infection is detected may be of value. Blanc et al. ${ }^{32}$ in 2015 reported that usage of targeted therapy with amoxicillin following positive UAT for pneumococcal pneumonia was associated with reduction of mortality. In the local setting, this may reduce the cost of using broad spectrum antibiotics and may lead to reduction of hospital stay. However, we should keep in mind that penicillin resistance rate in Streptococcus pneumoniae in Malaysia ranges from $21.2 \%$ in one study $^{33}$ up to $31.78 \%{ }^{34}$ in another.

\section{CONCLUSION}

In our study, the use of BinaxNOW kit increased the detection rate of pneumococcal pneumonia. We suggest greater education given to physicians on utility and value of BinaxNOW (or other validated direct antigen detection method). Since pneumococcal pneumonia was the commonest organism detected in our hospital and our initial empirical antibiotic of choice was appropriate, we suggest that for patients with severe pneumonia who are prescribed broad spectrum antibiotics, urinary BinaxNOW test should be performed with the intention to de-escalate antibiotic usage later. We suggest a follow up interventional, multicenter study to evaluate the impact of positive urinary Binax test or other similar validated test on deescalation of antibiotics. This will lead to cost savings and the reduction of antibiotic resistance.

\section{COMPETING INTEREST}

The authors declare that they have no competing interests

\section{LIST OF ABBREVIATIONS}

$\begin{array}{ll}\text { ATS } & \text { American Thoracic Society } \\ \text { CAP } & \text { Community-acquired Pneumonia } \\ \text { CRP } & \text { C-Reactive Protein } \\ \text { CVA } & \text { Cerebrovascular Accident } \\ \text { CXR } & \text { Chest Radiograph } \\ \text { ESR } & \text { Erythrocyte Sedimentation Rate } \\ \text { ETT } & \text { Endotracheal Tube } \\ \text { FBC } & \text { Full Blood Count } \\ \text { HCAP } & \text { Health-care Associated Pneumonia } \\ \text { IDSA } & \text { Infectious Disease Society of America } \\ \text { IQR } & \text { Inter-Quartile Range } \\ \text { LFT } & \text { Liver Function Test } \\ \text { MRSA } & \text { Methicillin Resistant Staphylococcus Aureus } \\ \text { OSAS } & \text { Obstructive Sleep Apnea Syndrome } \\ \text { PCR } & \text { Polymerase Chain Reaction } \\ \text { PTB } & \text { Pulmonary Tuberculosis } \\ \text { RP } & \text { Renal Profile } \\ \text { SPSS } & \text { Statistical Package for the Social Sciences } \\ \text { UAT } & \text { Urine Antigen Testing } \\ \text { UKMMC } & \text { University Kebangsaan Malaysia Medical Centre } \\ \text { UMMC } & \text { University Malaya Medical Center } \\ \text { US } & \text { United States }\end{array}$

\section{REFERENCES}

1. Curns AT, Holman RC, Sejvar JJ, Owings MF, Schonberger LB. Infectious disease

hospitalizations among older adults in the United States from 1990 through 2002. Arch Intern Med. 2005;165(21):2514-20.

2. Ruiz M, Ewig S, Marcos MA, Martinez JA, Arancibia F, Mensa J et al. Etiology of community-acquired pneumonia: impact of age, comorbidity, and severity. Am J Respir Crit Care Med. 1999;160(2):397-405.

3. Bates JH, Campbell GD, Barron AL, McCracken GA, Morgan PN, Moses EB et al. Microbial etiology of acute pneumonia in hospitalized patients. Chest. 1992;101(4):1005-12.

4. Greenberg D, Broides A, Blancovich I, Peled N, Givon-Lavi N, Dagan R. Relative importance of nasopharyngeal versus oropharyngeal sampling for isolation of Streptococcus pneumoniae and Haemophilus influenzae from healthy and sick individuals varies with age. J Clin Microbiol. 2004;42(10):4604-9.

5. Liam CK, Lim KH, Wong CM. Community-acquired pneumonia in patients requiring hospitalization. Respirology. 2001;6(3):259-64.

6. Hooi LN, Looi I, Ng AJ. A study on community acquired pneumonia in adults requiring hospital admission in Penang. Med J Malaysia. 2001;56 (3):275-84.

7. Mustafa MIA, Al-Marzooq F, How SH, Kuan YC, Ng $\mathrm{TH}$. The use of multiplex real-time PCR improves the detection of the bacterial etiology of community acquired pneumonia. Trop Biomed. 2011;28(3):531-44.

8. Community acquired pneumonia in Malaysia: Is Streptococcus pneumoniae an important pathogen? Niazlin M T, Andrea B Y, Roslina A M, 
Farida J and Ilina I. African Journal of Microbiology Research Vol. 6(3), pp. 512-519, 23 January, 2012.

9. Lim WS, Macfarlane JT, Boswell TC, Harrison TG, Rose D, Leinonen M et al. Study of community acquired pneumonia aetiology (SCAPA) in adults admitted to hospital: implications for management guidelines. Thorax. 2001;56(4):296-301.

10. Murdoch DR, Laing RT, Mills GD, Karalus NC, Town GI, Mirrett $S$ et al. Evaluation of a rapid immunochromatographic test for detection of Streptococcus pneumoniae antigen in urine samples from adults with community-acquired pneumonia. J Clin Microbiol. 2001;39(10):34958.

11. Kollef MH, Shorr A, Tabak YP, Gupta V, Liu LZ, Johannes RS. Epidemiology and outcomes of health-care-associated pneumonia: results from a large US database of culture-positive pneumonia. Chest. 2005;128(6):3854-62.

12. Micek ST, Kollef KE, Reichley RM, Roubinian N, Kollef $\mathrm{MH}$. Health care-associated pneumonia and community-acquired pneumonia: a singlecenter experience. Antimicrob Agents Chemother. 2007;51(10):3568-73.

13. Venditti M, Falcone M, Corrao S, Licata G, Serra $P$, Study Group of the Italian Society of Internal Medicine. Outcomes of patients hospitalized with community-acquired, health careassociated, and hospital-acquired pneumonia. Ann Intern Med. 2009;150(1):19-26.

14. Shindo Y, Sato S, Maruyama E, Ohashi T, Ogawa $\mathrm{M}$, Hashimoto $\mathrm{N}$ et al. Health-care-associated pneumonia among hospitalized patients in a Japanese community hospital. Chest. 2009;135 (3):633-40.

15. Andreo F, Domínguez J, Ruiz J, Blanco S, Arellano E, Prat $C$ et al. Impact of rapid urine antigen tests to determine the etiology of community-acquired pneumonia in adults. Respir Med. 2006;100(5):884-91.

16. Lauren K. Troy, Keith K.H. Wong, David J. Barnes. Prevalence and Utility of Positive Pneumococcal Urinary Antigen Tests in Australian Patients with Community-Acquired Pneumonia. ISRN Infectious Disease. 2013, Article ID 518205, 5 pages.

17. Charles PGP, Whitby M, Fuller AJ, Stirling R, Wright AA, Korman TM et al. The etiology of community-acquired pneumonia in Australia: why penicillin plus doxycycline or a macrolide is the most appropriate therapy. Clin Infect Dis. 2008;46(10):1513-21.

18. Wattanathum A, Chaoprasong C, Nunthapisud P, Chantaratchada S, Limpairojn N, Jatakanon A et al. Community-acquired pneumonia in southeast Asia: the microbial differences between ambulatory and hospitalized patients. Chest. 2003;123(5):1512-9.

19. Reechaipichitkul W, Lulitanond V, Sawanyawisuth K, Lulitanond A, Limpawattana P. Etiologies and treatment outcomes for outpatients with community-acquired pneumonia
(CAP) at Srinagarind Hospital, Khon Kaen, Thailand. Southeast Asian J Trop Med Public Health. 2005;36(5):1261-7.

20. Bansal S, Kashyap S, Pal LS, Goel A. Clinical and bacteriological profile of community acquired pneumonia in Shimla, Himachal Pradesh. Indian J Chest Dis Allied Sci. 2004;46 (1):17-22.

21. Saito A, Kohno S, Matsushima T, Watanabe A, Oizumi K, Yamaguchi K et al. Prospective multicenter study of the causative organisms of community-acquired pneumonia in adults in Japan. J Infect Chemother. 2006;12(2):63-9.

22. Kobashi Y, Yoshida K, Miyashita N, Niki Y, Matsushima T. Evaluating the use of a Streptococcus pneumoniae urinary antigen detection kit for the management of community-acquired pneumonia in Japan. Respiration. 2007;74(4):387-93.

23. Ishida $T$, Hashimoto $T$, Arita $M$, Tojo $Y$, Tachibana $H$, Jinnai $M$. A 3-year prospective study of a urinary antigen-detection test for Streptococcus pneumoniae in communityacquired pneumonia: utility and clinical impact on the reported etiology. J Infect Chemother. 2004;10(6):359-63.

24. Domínguez J, Galí N, Blanco S, Pedroso P, Prat C, Matas L et al. Detection of Streptococcus pneumoniae antigen by a rapid immunochromatographic assay in urine samples. Chest. 2001;119(1):243-9.

25. Marcos MA, Jiménez de Anta MT, de la Bellacasa JP, González J, Martínez E, García E et al. Rapid urinary antigen test for diagnosis of pneumococcal community-acquired pneumonia in adults. Eur Respir J. 2003;21 (2):209-14.

26. Gutiérrez F, Masiá M, Rodríguez JC, Ayelo A, Soldán B, Cebrián L et al. Evaluation of the immunochromatographic Binax NOW assay for detection of Streptococcus pneumoniae urinary antigen in a prospective study of communityacquired pneumonia in Spain. Clin Infect Dis. 2003;36(3):286-92.

27. Smith MD, Derrington $P$, Evans R, Creek M, Morris R, Dance DAB et al. Rapid diagnosis of bacteremic pneumococcal infections in adults by using the Binax NOW Streptococcus pneumoniae urinary antigen test: a prospective, controlled clinical evaluation. J Clin Microbiol. 2003;41(7):2810-3.

28. Priner M, Cornillon C, Forestier D, Valero S, Paccalin M. Might Streptococcus pneumoniae urinary antigen test be positive because of pneumococcal vaccine? J Am Geriatr Soc. 2008;56(1):170-1.

29. Kelley R, Keyserling $H$. Antigen excretion in the urine after pneumococcal vaccination. Pediatr Infect Dis J. 1997;16(7):720.

30. Boulware DR, Daley CL, Merrifield C, Hopewell PC, Janoff EN. Rapid diagnosis of pneumococcal pneumonia among HIV-infected 
adults with urine antigen detection. J Infect. 2007;55(4):300-9.

31. Arushothy R, Ahmad N, Md Yassin R. Evolution of Erythromycin Resistance among

Streptococcus pneumoniae Isolates in Malaysia from 2005 and 2010. Journal of Biosciences and Medicines. 2016;4:116-22.

32. Blanc V, Mothes A, Smets A, Timontin I, Guardia MD, Billiemaz A et al. Severe community acquired pneumonia and psitive urinary antigen test for

S.pneumoniae:amoxicillin is associated with favourable outcome. Our J Clin Microbial Infect Dis. 2015;34(12):2455-61.

33. Le C-F, Palanisamy NK, Mohd Yusuf MY, Sekaran SD (2011). Capsular Serotype and antibiotic esistance of Streptococcus pneumoniae Isolates in Malaysia. PLoS ONE 6(2011), p.e1954732

34. Yasin RM, Zin NM, Hussin A, Nawi SH, Hanapiah SM, Wahab ZA et al. Current trend of pneumococcal serotypes distribution and antibiotic succesptibility pattern in Malaysian hospitals. Vaccine. 2011;29(34):5688-93. 